\title{
Circuit
}

Musiques contemporaines

\section{Introduction : harmonie préétablie ?}

\section{Jonathan Goldman}

Volume 22, numéro 1, 2012

Arts de la synchronisation

URI : https://id.erudit.org/iderudit/1008964ar

DOI : https://doi.org/10.7202/1008964ar

Aller au sommaire du numéro

Éditeur(s)

Les Presses de l’Université de Montréal

ISSN

1183-1693 (imprimé)

1488-9692 (numérique)

Découvrir la revue

Citer ce document

Goldman, J. (2012). Introduction : harmonie préétablie ? Circuit, 22(1), 5-8.

https://doi.org/10.7202/1008964ar d'utilisation que vous pouvez consulter en ligne.

https://apropos.erudit.org/fr/usagers/politique-dutilisation/ 


\section{Introduction : Harmonie préétablie?}

Jonathan Goldman

Pour comprendre le mécanisme de coordination entre l'âme et le corps, Leibniz a revisité la théorie dite occasionaliste du philosophe rationaliste Nicolas Malebranche. Le philosophe allemand a postulé que les substances corporelles et spirituelles ne sont en réalité jamais dans un rapport de cause à effet, mais que cette illusion s'observe grâce à un dispositif divin qu'il baptisait «l'harmonie préétablie». Cette doctrine qui attribue à Dieu le rôle de synchronisateur du corps et de l'âme n'est pas sans rapport avec le thème de ce numéro qui explore les diverses façons dont la musique et d'autres sphères d'activités humaines peuvent marcher de pair pourvu que certaines conditions soient préalablement remplies. Il sera donc question de synchronisation, un thème que Philippe Despoix, professeur de littérature comparée à l'Université de Montréal et directeur de la revue Intermédialités, a notamment exploré dans ses recherches sur l'orientation cartographique puis à propos des différents systèmes d'écriture, et qu'il poursuit maintenant du côté du cinéma et d'autres médias'. C'est d'ailleurs Philippe Despoix qui nous a suggéré de «synchroniser » - à quelques mois près - ce numéro de Circuit et le numéro de printemps 2012 d'Intermédialités avec, dans chacun d'eux, un dossier consacré aux questions de synchronisation. La revue Circuit est donc ravie de s'associer à l'équipe d'Intermédialités, même si c'est au comité de rédaction de Circuit que revient tout le crédit - et tout le blâme cas échéant! - pour son contenu.

Dans le dossier thématique de ce numéro, nous explorons donc différentes façons dont la synchronisation entre dans le cercle des préoccupations des musiciens. Dès lors que la musique s'associe à d'autres arts qui se déploient dans le temps, comme la danse, le théâtre ou le cinéma, la synchronisation devient un enjeu inéluctable. Différentes approches se sont imposées au cours du $\mathrm{Xx}^{\mathrm{e}}$ siècle pour coordonner deux médias en simultané; il y a l'approche que l'on rencontre parfois au cinéma où la musique est subordon-
1. Voir par exemple Philippe Despoix, Le Monde mesuré: dispositifs de l'exploration à l'âge des Lumières, Genève, Droz, 2005; "Questions et hypothèses à partir des systèmes d'écritures: remédiation ou plurimédialité?", Intermédialités. Histoire et théories des arts, des lettres et des techniques, $n^{\circ} 6$, 2005, p. 95-106; "Le médium technique: objet et $a$ priori. Archive phonographique et musicologie comparée", Revue de Synthèse, $n^{\circ}$ 3, 2008, p. 341-361. 
2. Voir The Carl Stalling Project: Music From Warner Bros. Cartoons, 1936-1958, Warner Bros., 1990.

3. Voir par exemple le spectacle Doubles points: OYTI, créé le 28 janvier 2011 à la Cité de la musique à Paris. Une autre tentative de fusion entre le geste du musicien et celui du danseur se trouve dans la mise en scène signée Xavier Le Roy de Salut für Caudwell, pour guitare, de Helmut Lachenmann. Un guitariste interprète l'œuvre derrière un rideau alors qu'un autre interprète mime les gestes du guitariste sur scène - mais sans guitare - en restant en parfaite synchronisation avec ce dernier, créant un dispositif non loin de ce qui se nomme communément le "Air Guitar" (voir par exemple, www. airguitarworldchampionships.com/en/ event/agwc-2012), certainement une manifestation populaire incontournable de la synchronisation entre le geste et le son. Voir Xavier Le Roy, "Staging Salut für Caudwell (Preliminary Notes and Retrospective Questions)", entretien réalisé par Nicolas Donin, L'inouï, Revue de l'Ircam, no 2 (2006), inoui.ircam. $\mathrm{fr} / 596 . \mathrm{html}$ ?L=1

4. Un tableau particulièrement évocateur de la démarche de Cunningham est dépeint par Carolyn Brown dans ses mémoires récents Chance and circumstance: twenty years with Cage and Cunningham, New York: Alfred A. Knopf, 2007. Voir aussi, Roger Copeland, Merce Cunningham: the modernizing of modern dance, New York, Routledge, 2004, et le nouveau coffret Music for Merce: 1952-2009, New World Records, 80712, 2010, 10 CD.

5. Par exemple dans le mouvement Putnam's Camp de Three Places in New England (1912). née à l'action, où, par exemple, une balle lancée est l'occasion d'un glissando à la clarinette; cette approche est si fortement associée aux bandes sonores de dessins animés qu'on l'appelle couramment en anglais le «MickeyMousing» en l'honneur du célèbre protagoniste des dessins animés de Walt Disney, frénétiquement mis en musique par des compositeurs tels que Carl Stalling². Des collaborations récentes entre le chorégraphe italo-néerlandais Emio Greco et le compositeur suisse Hanspeter Kyburz favorisent aussi le synchrone - même si leur idiome stylistique ne doit rien à celui du dessin animé! - en ce que des gestes du danseur sont captés et analysés pour ensuite influencer le cours de la musique (électronique)3. Aux antipodes du MickeyMousing se trouve l'approche chorégraphique du récemment disparu Merce Cunningham (1919-2009), qui préconise un art pour ainsi dire bi-temporel où danse et musique restent à dessein parfaitement asynchrones, au point où ils se rencontrent parfois pour la première fois au moment du spectaclet.

Toutefois, nul besoin d'évoquer la danse ou le cinéma alors qu'une problématique de la synchronisation s'observe au sein même de la musique. Dès lors que deux musiciens jouent de et en concert il leur faut s'entendre sur une méthode pour placer leurs différentes lignes musicales dans une seule et même trame. Or, dans la musique de concert traditionnelle, la synchronisation des différentes parties est assurée par le chef d'orchestre. Cette situation classique s'est vue élargie au cours du Xxe siècle par différents dispositifs plus complexes: il y a la musique polymétrique de Charles Ives, où plusieurs groupes instrumentaux jouent ensemble mais à différentes vitesses, nécessitant deux chefs d'orchestre ou un seul capable de battre deux tempi différents (un tempo différent avec chacun de ses bras)5; dans une œuvre comme Jeux vénitiens (1960-1961) de Witold Lutosławski (1913-1994), le compositeur laisse au hasard la synchronisation des parties individuelles, sans pour autant renoncer à ses responsabilités quant à l'articulation de la forme globale. Il a nommé cette technique, qui implique un transfert (limité) de pouvoir du chef aux instrumentistes, «l'aléatoire contrôlé». Dans le texte d'une conférence fondamentale de 1962, publiée ici dans la rubrique Document dans une traduction française inédite, Lutosławski précise que dans une telle œuvre «la rythmique, étant la somme de toutes les parties, ne peut pas être prévue en détail, néanmoins sa physionomie générale est largement tributaire de la rythmique de chaque partition individuelle, et donc de la volonté du compositeur».

D’autres expériences musicales ont pourtant précédé ces réalisations, qui repensaient la synchronisation parfois de manière encore plus radicale: l'orchestre soviétique Persimfans, fondé par Lev Tseitlin, s'est voué dans les 
années 1920 à l'interprétation du répertoire classique sans l'aide d'un chef d'orchestre, dans un esprit d'égalitarisme ${ }^{6}$. Cette tentative a notamment inspiré un projet semblable en Allemagne, le Leipziger Sinfonie-Orchester ohne Dirigenten, fondé en 19287 . Plus près de nous, le compositeur francoslovène Vinko Globokar (1934) a aussi réalisé, dans un esprit de l'après-1968, son projet Das Orchester (1974) qui se dispense d'un chef. Fasciné par la psychologie de la foule après la lecture d'Elias Canetti ${ }^{8}$, il compose Masse Macht und Individuum (1995) qui explore le rapport entre l'individu et le groupe, symbolisé par l'orchestre. Il retourne à la formule de l'orchestre «décapité» dans Les chemins de la liberté de 2003-2004, où l'instrumentiste principal de chacun des six groupes instrumentaux se tourne vers sa section, et bat, à l'aide d'un métronome, la mesure de son groupe, différente de celles des autres groupes?.

Allier le son électronique au son instrumental reste bien sûr une des préoccupations durables de l'Ircam, cet institut de recherche et de coordination acoustique/musique qui demeure depuis sa fondation au milieu des années 1970, un chef de file de la recherche sur les interactions en temps réel. Arshia Cont, réalisateur musical à l'Ircam, dresse un bilan des travaux effectués dans le domaine du synchronisme dans les musiques mixtes (c'està-dire pour instruments de musique et dispositif électronique). Pour sa part, le compositeur Andrew Culver, ancien assistant (notamment en matière d'informatique musicale) de John Cage et défenseur passionné de l'orchestre sans chef, corrollaire de ce qu'il nomme « anarchic harmony $»^{10}$, se livre à des réflexions qui débordent le cadre de la musique, et montre au passage que l'orchestre sans chef pourrait inspirer toute association de personnes vouées à un but commun. Barah Héon-Morissette témoigne de l'expérience d'un spectacle conçu par le compositeur et cinéaste Thierry de Mey qui lie son et lumière de façon inouïe.

La compositrice / interprète Cléo Palacio-Quintin résume ses recherches avec l'instrument dont elle joue depuis bientôt quinze ans, son hyper-flûte autofabriquée, qui permet de multiples axes de contrôle sur un dispositif électronique en direct dans un contexte de performance. Par ailleurs, nous sommes ravis d'accueillir Cléo non plus seulement comme membre du comité de rédaction, mais désormais en tant que directrice de la rubrique Actualités. Quant à cette rubrique, outre des comptes-rendus de livres, de disques et de concerts, vous y trouverez une nouvelle chronique s'intitulant Entendu dans Cette ville étrange, réalisée par l'équipe du site internet Cette ville étrange (www.cettevilleetrange.org). Dans la première livraison, Michel Gonneville se penche sur le catalogue du jeune compositeur Julien Bilodeau. En outre,
6. Voir Eckhard John, "OrchesterRevolution. Das dirigentenlose Orchester "Persimfans" (Moskau 19221933)", in Musik/Revolution. Festschrift zum 90. Geburtstag von Georg Knepler, vol. 2, Hanns-Werner Heister (dir.), Hamburg, Von Bockel 1997, p. 259-277.

7. Eckhard John, "Orchester ohne Dirigent (II) : Die dirigentenlosen Konzerte in Leipzig (1928/1929)", Das Orchester, Zeitschrift für Orchesterkultur und Rundfunk-Chorwesen, no 1 (1998), p. 11-15.

8. Elias Canetti, Masse et puissance (1960), trad. Robert Rovini, Paris, Gallimard, 1966.

9. Sur Globokar, voir brahms.ircam. fr/vinko-globokar ainsi que John Warnaby, "Vinko Globokar: Revaluing a phenomenon", Tempo, vol. 61, $n^{\circ} 240$ (2007), p. 2-18. Explication du fonctionnement extrait d'un entretien avec Vinko Globokar réalisé par l'auteur à Toronto le 7 décembre 2011.

10. Voir son site: www.anarchicharmony.org/ 
11. Voir une collaboration Messier/ Bernier (prix2011.aec.at/winner/222/). Une installation sonore de Bellavance peut être visionnée ici : vimeo. com/30626467

12. Voir Intermédialités: www.erudit. org/revue/im/apropos.html. Le paragraphe qui suit a été rédigé avec le concours de Philippe Despoix.

13. Voir entre autres Michel Chion, Un art sonore, le cinéma : histoire, esthétique, poétique, Paris: Cahiers du cinéma - Essais, 2003.

14. Voir Anette Rose, Enzyklopädie der Handhabungen 2006-2010, BielefeldLeipzig-Berlin, Kerber Art, 2010 non sans rapport avec la synchronisation, dans le Cahier d'analyse, FrançoisHugues Leclair nous invite dans l'atelier de Michel Longtin, un compositeur de musique de concert qui s'inspire de la musique de film, tirant des leçons insolites de Jerry Goldsmith ou John Barry. Enfin, les illustrations qui ornent ce numéro documentent la démarche de l'artiste sonore Martin Messier, qui, tout comme ses collègues Nicolas Bernier et Alexis Bellavance, réalise des spectacles où le son est issu de machines trouvées ou de sa propre construction $^{11}$

Conformément à la vocation interdisciplinaire d'une revue qui se consacre à l'histoire et aux théories des arts, des lettres et des techniques, le nº 19 d'Intermédialités, «synchroniser / synchronize », coordonné par Philippe Despoix et Nicolas Donin (Ircam) abordera ce thème sous l'angle le plus large ${ }^{12}$. Partant de l'idée que tout type de technique autorise ou induit des effets de (dé)synchronisation, ce numéro explorera en premier lieu les systèmes d'écriture comme mise en relation entre le langage, la pensée, le corps et la lecture. Il s'intéressera aussi aux questions musicales en interrogeant la mutation des pratiques orchestrales au XIX siècle mais également la manière dont la coordination paralinguistique peut s'articuler, au $\mathrm{Xx}^{\mathrm{e}}$, avec l'improvisation en jazz, ou encore en rappelant les rares essais de synchronisation entre animation audiovisuelle et musique concrète. L'enquête se penchera de plus vers les rapports de synchronie possibles entre son, geste et image: que ce soit sur la scène théâtrale ou, à travers le corps même de l'acteur, au cinéma - ce média permettant de revenir sur la notion de «synchrèse ${ }^{13}$ » ainsi que sur les modes de (dis)jonction par lesquels le regard et l'écoute peuvent frayer leur voie l'un vis-à-vis de l'autre. Pour conclure, un dossier visuel donnera l'occasion à un artiste vidéaste de mettre en perspective, par le biais de juxtapositions, des opérations manuelles de synchronisation accompagnant certains processus machinaux traditionnels ${ }^{14}$.

Enfin, signalons la contribution considérable voire décisive de John Rea qui quitte le comité de rédaction de la revue après 22 ans de service. Membre fondateur, John a fait preuve d'un investissement personnel hors pair. Il a signé treize articles dans autant de numéros, et il a laissé ainsi son empreinte indélébile sur Circuit, par sa plume fine, intelligente, et parfois - oserais-je dire - ironique. Nous comptons sur John Rea pour enrichir Circuit de nouveaux articles, et je saisis cette occasion pour le remercier sincèrement.

Bonne lecture!

Montréal, avril 2012 